\title{
Chemical potential response of meson masses at finite temperature
}

\author{
QCD-TARO Collaboration: Ph. de Forcrand ${ }^{\mathrm{a}} \mathrm{b}, \mathrm{T}$. Hashimoto $^{\mathrm{c}}, \mathrm{S} . \mathrm{Hioki}^{\mathrm{d}}$, Y. Liu ${ }^{\mathrm{e}}, \mathrm{H}$. Matsufuru $^{\mathrm{f}}$, \\ O. Miyamura ${ }^{\mathrm{e}}$, A. Nakamura ${ }^{\mathrm{g}}$, T. Takaishi ${ }^{\mathrm{h}}$ and T. Umeda ${ }^{\mathrm{e}}$ \\ anst. für Theoretische Physik, ETH-Hönggerberg, CH-8093 Zürich, Switzerland \\ ${ }^{\mathrm{b}}$ CERN, Theory Division, CH-1211 Geneva 23, Switzerland \\ ${ }^{\mathrm{c}}$ Department of Applied Physics, Faculty of Engineering, Fukui University, Fukui 910-8507, Japan \\ ${ }^{\mathrm{d}}$ Department of Physics, Tezukayama University, Nara 631-8501, Japan \\ e Department of Physics, Hiroshima University, Higashi-Hiroshima 739-8526, Japan \\ ${ }^{\mathrm{f}}$ Research Center for Nuclear Physics, Osaka University, Ibaraki 567-0047, Japan \\ g Research Institute for Information Science and Education, Hiroshima University, Higashi-Hiroshima \\ 739-8521, Japan \\ ${ }^{\mathrm{h}}$ Hiroshima University of Economics, Hiroshima 731-01, Japan
}

\begin{abstract}
We study the response of meson masses to the chemical potential $(\partial m / \partial \mu)$ at high temperature and at zero chemical potential on $N_{f}=2$ lattice with staggered fermions. Preliminary results for the meson composed of different quarks show that $\partial m /\left.\partial \mu\right|_{\mu=0}$ is negative in the confinement phase and positive in the deconfinement phase.
\end{abstract}

\section{INTRODUCTION}

The temperature and density effects to the properties of hadrons are interesting and important for the early universe and for high energy physics. So far the temperature effect has been extensively studied [1]. But there are not so many results concerning the density effect. As is well known, there are difficulties with the simulation of a finite density system by the lattice QCD approach. The reason is that the fermionic determinant becomes complex and cannot be considered as a part of the probability. If we factor out the phase of the determinant and put it into observables, it leads to oscillating contributions in quantum averages. This makes it hard to obtain reliable results. It has been also known that the naive quenched approximation leads to an essentially different world [2].

In spite of this difficult situation, density effects to hadrons such as a mass-shift are very in- teresting and important subjects both theoretically and experimentally [3,4]. There are several approaches to circumvent this difficulty and they seem successful to a limited extent [5]. Another way is to study two-color QCD [6 11]. Also, a non-trivial quenched approximation can be defined [12] by taking the simultaneous limit where the quark mass and the logarithm of the chemical potential both become infinite 13$]$. As for the response to the chemical potential, only the baryon number susceptibility at zero baryon density has been studied, and an abrupt jump at the transition point has been reported [14].

In this paper, we examine the response to the chemical potential of the mass of hadrons at finite temperature and at zero baryon density in full $\mathrm{SU}(3)$ QCD simulations 15. 


\section{CHEMICAL POTENTIAL RE- SPONSE OF HADRONIC MASSES AT HIGH TEMPERATURE}

As stated in the introduction, the lattice study at finite density is still difficult. In this situation, an interesting possibility is to examine the response of physical quantities to the chemical potential at zero chemical potential.

Here we consider a hadronic correlator $G(x)$,

$G(x)=\sum_{y, z, t}<H(x, y, z, t) H(0,0,0,0)^{\dagger}>$,

where $H(x, y, z, t)$ denotes the hadron operator.

Suppose that this hadronic correlator is dominated by a single pole, then

$G(x) \approx A e^{-m x}$.

We take derivative with respect to chemical potential $\mu$,

$$
\begin{aligned}
B(x) & \equiv G(x)^{-1} \frac{\partial G(x)}{\partial \mu} \\
& =A^{-1} \frac{\partial A}{\partial \mu}-\frac{\partial m}{\partial \mu} x .
\end{aligned}
$$

In eq.(14), if the left hand side is measured as a function of $x$, the linear term gives the chemical potential response of the hadron mass, while the constant term gives the response of the coupling.

The next problem is to get the derivative of the correlator. For this purpose, we go back to the definition of the hadronic correlator.

$$
\begin{aligned}
& <H(n) H(0)^{\dagger}>= \\
& Z^{-1} \int[d U] \operatorname{Tr}\left(M(n ; 0) \Gamma M(0 ; n) \Gamma^{\dagger}\right) \\
& \operatorname{det}(D) \exp \left(-S_{G}\right)
\end{aligned}
$$

where $Z=\int[d U] \operatorname{det}(D) \exp \left(-S_{G}\right)$ and $M=$ $D^{-1}$.

Then, using the formulae

$\frac{\partial \operatorname{det}(D)}{\partial \mu}=\operatorname{Tr}\left(M \frac{\partial D}{\partial \mu}\right) \operatorname{det}(D)$

and

$\frac{\partial M}{\partial \mu}=-M \frac{\partial D}{\partial \mu} M$, we get

$$
\begin{aligned}
& \frac{\partial<H(n) H(0)^{\dagger}>}{\partial \mu}= \\
&-\quad<\operatorname{Tr}\left(M \dot{D} M \Gamma M \Gamma^{\dagger}\right)> \\
&-\quad<\operatorname{Tr}\left(M \Gamma M \dot{D} M \Gamma^{\dagger}\right)> \\
&+\quad<\operatorname{Tr}\left(M \Gamma M \Gamma^{\dagger}\right) \operatorname{Tr}(\dot{D} M)> \\
&-\quad<\operatorname{Tr}\left(M \Gamma M \Gamma^{\dagger}\right)><\operatorname{Tr}(\dot{D} M)>
\end{aligned}
$$

where the short hand notations

$\dot{X}=\frac{\partial X}{\partial \mu}$

and

$$
<Y>=Z^{-1} \int[d U] \operatorname{det}(D) \exp \left(-S_{G}\right) Y
$$

are used. Here we calculate the first and second terms of the right hand side of eq.(8) and ignore the other terms. $\operatorname{Tr}(\dot{D} M)$ is pure imaginary and its average value, i.e. $<\operatorname{Tr}(\dot{D} M)>$ will be zero 14. If $\operatorname{Tr}\left(M \Gamma M \Gamma^{\dagger}\right)$ is real, the third term does not contribute as real and we may neglect it. For degenerate quarks, $\operatorname{Tr}\left(M \Gamma M \Gamma^{\dagger}\right)$ is real. Although we consider here non-degenerate systems for which $\operatorname{Tr}\left(M \Gamma M \Gamma^{\dagger}\right)$ is not real, we assume that the contribution from the third term is small and drop it in this study.

We introduce two independent chemical potentials, $\mu_{q}$ and $\mu_{Q}$ for two different quarks ( $\left.m_{q} \leq m_{Q}\right)$. We introduce convenient definitions of the chemical potential, $\mu_{s} \equiv\left(\mu_{q}+\mu_{Q}\right) / 2$ and $\mu_{v} \equiv\left(\mu_{q}-\mu_{Q}\right) / 2$ and define the following derivatives with respect to $\mu_{s}$ and $\mu_{v}$.

$$
\begin{aligned}
& \frac{\partial}{\partial \mu_{s}}=\frac{\partial}{\partial \mu_{q}}+\frac{\partial}{\partial \mu_{Q}} \\
& \frac{\partial}{\partial \mu_{v}}=\frac{\partial}{\partial \mu_{q}}-\frac{\partial}{\partial \mu_{Q}}
\end{aligned}
$$

$\mu_{s}$ is usual chemical potential corresponding to baryon number. In this study we consider the response with respect to $\mu_{s}$.

In the case of the staggered fermion formalism, the derivative of the fermion operator is

$$
\begin{aligned}
& \frac{\partial D_{K S}}{\partial \mu}= \\
& \frac{a}{2}\left[U_{t}(n) e^{a \mu} \delta_{n+t, m}\right. \\
+\quad & \left.U_{t}^{\dagger}(n-t) e^{-a \mu} \delta_{n-t, m}\right] .
\end{aligned}
$$


At $\mu=0$, we can evaluate eqs. (5) and (8) by usual techniques of lattice QCD simulation.

\section{PRELIMINARY RESULTS}

Here we present our preliminary result for $\partial m /\left.\partial \mu\right|_{\mu=0}$.

\subsection{Lattice parameters}

Lattice parameters are as follows:

\section{- Lattice Size:}

$16 \times 8 \times 8 \times 4$, with 16 for $x$ direction, 4 for the temperature direction.

\section{- Quarks:}

$m_{Q}=0.25$ and $m_{q}=0.025$ for $\beta=5.26$, and $m_{Q}=0.25$ and $m_{q}=0.0125$ for $\beta=5.33$. Dynamical K-S fermions with $N_{f}=2$ are the light quarks $\left(m_{q}\right)$. The meson operator, $H$, is made of the two different quarks.

\section{- Temperature:}

$\beta=5.26$ for $T \approx 0.97 T_{c}$ (confinement), and $\beta=5.33$ for $T \approx 1.07 T_{c}$ (deconfinement).

\section{- Statistics:}

40-90 configurations for each parameter.

\section{2. $\partial m /\left.\partial \mu_{s}\right|_{\mu=0}$}

The results for the $B(x)$ in eq.(3) are shown in Fig. 1 and Fig.2. Fig. 1 is for $\beta=5.26$ which corresponds to the confinement phase and Fig.2 is for $\beta=5.33$ which corresponds to the deconfinement phase.

As is stated above, the coefficient of the linear term in $B(x)$ corresponds to $-\partial m /\left.\partial \mu\right|_{\mu=0}$. Although this is preliminary and the statistics are small, we can see that $\partial m /\left.\partial \mu\right|_{\mu=0}$ is negative in the confinement phase and $\partial m /\left.\partial \mu\right|_{\mu=0}$ is positive in the deconfinement phase.

\section{4. $J / \psi$ SUPPRESSION WITHOUT QGP}

One of our masses, $m_{Q}$, is in the region of the strange quark. If the behaviors observed in Figs. 1 and 2 are also seen in the charm quark mass region, then the mass of the heavy-light meson, $D$, might decrease at very small chemical potential just below the deconfinement temperature. Then

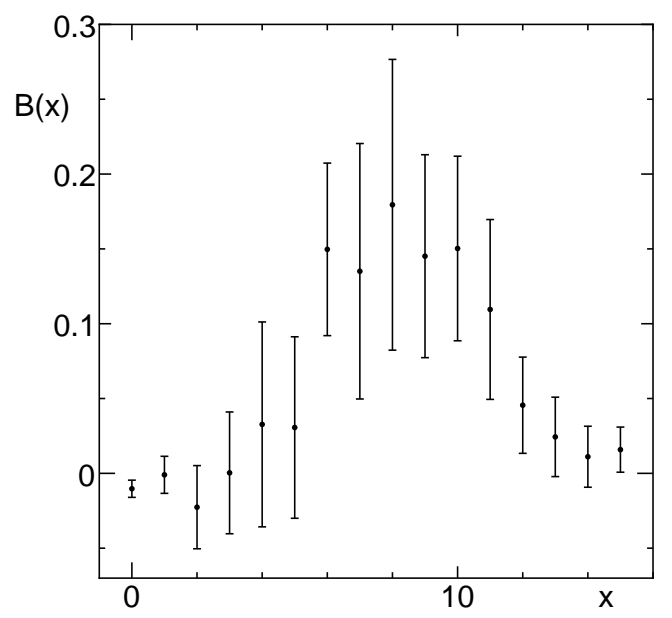

Figure 1. $\quad G(x)^{-1} \frac{\partial G(x)}{\partial \mu_{s}}{ }_{\mu=0}$ in the confinement phase $\left(\beta=5.26, T \approx 0.97 T_{c}\right)$, where $G(x)$ is the hadron propagator along the $x$ direction. $m_{Q}=$ 0.25 and $m_{q}=0.025$

the mass of $D \bar{D}$ system might be lower than the mass of the $\psi^{\prime}$ meson, and a new decay mode

$\psi^{\prime} \rightarrow D+\bar{D}$

will open.

If this happens in, for example, ultrarelativistic heavy ion collisions, $\psi^{\prime}$ mesons produced by this collision will decay as $\psi^{\prime} \rightarrow D+$ $\bar{D}$ and eventually the amount of $J / \psi$ produced through $\psi^{\prime} \rightarrow \chi \rightarrow J / \psi$ channel will decrease. This means that $J / \psi$ suppression might occur even in the confinement phase as suggested by Hayashigaki[16].

\section{SUMMARY}

The results of this paper are very preliminary and qualitative and should be checked in the near future. However a model calculation based on NJL model[17] suggests the same tendency. 


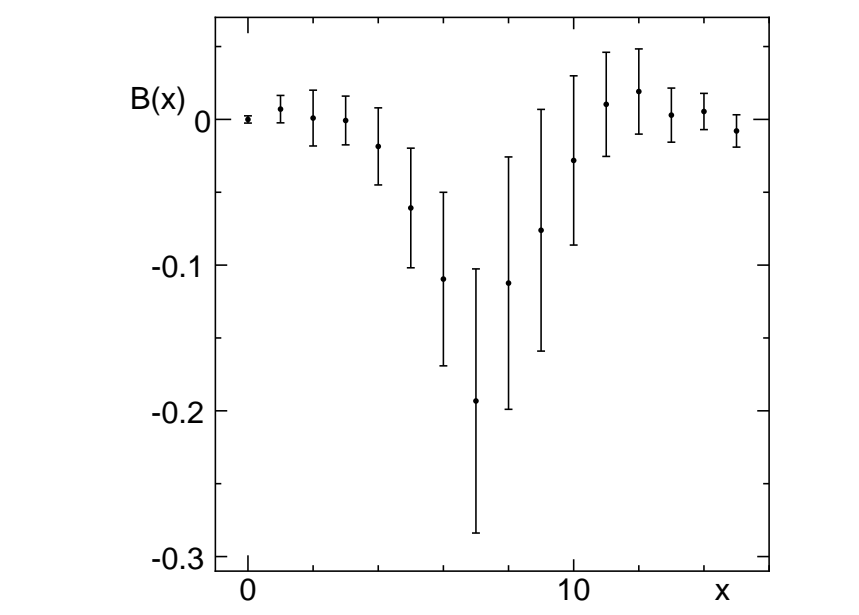

7. M.-P. Lombardo, hep-lat/9907025, heplat/9906006.

8. S. Hands, J. B. Kogut, M-P. Lombardo and S. E. Morrison, Nucl. Phys. B558 (1999) 327.

9. S. Morrison and S. Hands, hep-lat/9902012.

10. S. Hands et al., hep-lat/0006018.

11. S. J. Hands, J. B. Kogut, S. E. Morrison, D. K. Sinclair, hep-lat/0010028.

12. J. Engels, O. Kaczemarek, F. Karsch, E. Laermann, Nucl. Phys. B558 (1999) 307.

13. I.Bender, T.Hashimoto, F.Karsch, V.Linke, A.Nakamura, M.Plewnia, I.O.Stamatescu and W.Wetzel, Nucl.Phys. B(Proc.Suppl.)26 323 (1992); T.C.Blum, J.E.Hetrick and D.Toussaint, Phys. Rev. Lett. 761019 (1996).

14. S. Gottlieb et al., Phys. Rev. D55 6852 (1997)

Figure 2. $G(x)^{-1} \frac{\partial G(x)}{\partial \mu_{s}} \mu=0$ in the deconfinement phase $\left(\beta=5.33, T \approx 1.07 T_{c}\right)$, where $G(x)$ is the hadron propagator along the $x$ direction. $m_{Q}=$ 0.25 and $m_{q}=0.0125$

\section{ACKNOWLEDGMENT}

We thank I. O. Stamatescu for a critical reading of the manuscript. This work is partially supported by the Grant-in-Aide for Scientific Research by JSPS and Monbusho, Japan (No. 10640272, No. 11440080, No. 11694085, No. 11740159 and No. 12554008).

\section{REFERENCES}

1. See for example: The proceedings of "Quark Matter" and "Lattice Field Theory" conferences.

2. M.A. Stephanov, Phys.Rev.Lett. 764472 (1996).

3. T. Hatsuda and S.H. Lee, Phys. Rev. C46 34 (1992).

4. G. Agakishiev et al., CERES collaboration, Nucl. Phys. A638 159 (1998).

5. I.M. Barbour, S.E. Morrison, E.G. Klepfish, J.B. Kogut and M. Lombardo, Nucl.Phys. B(Proc.Suppl.)60A 220 (1998).

6. A. Nakamura, Phys. Lett., 149B (1984) 391.

15. See also Ph. de Forcrand et al., QCDTAROcollaboration , Nucl.Phys. B(Proc.Suppl.)73 477 (1999).

16. A. Hayashigaki, "New Directions in Quantum Chromodynamics", ed. by C-R. Ji and D-P. Min, AIP Conference Proceedings 494, Melville, New York, 1999, p429.

17. See T. Hatsuda and T. Kunihiro, Phys. Rep. C247 221 (1994). 